\title{
XIII.
}

\section{Die Tetanie.}

Eine ätiologisch-pathologische Studie.

\author{
Von
}

\section{Dr. Arthur Sarb6,}

Nervenarzt in Budapest.

Im Frühjahr hatte ich Gelegenheit, drei Fälle von Tetanie zu sehen, von denen zwei, sich auf eine Mutter und ihr Kind bezogen. Die gründliche klinische Aufnahme der Fälle scheiterte an äusseren Verhältnissen. Die Mutter, 30 Jahre alt, Tagelöhnerin, erkrankte ohne nachweisbare Ursache an in beiden Händen eintretenden mit Schmerzen verbundenen Contracturen. Die Hände sind im Handgelenk flectirt, die Finger extendirt und im Metacarpophalangealgelenk flectirt; der Daumen an den Zeigefinger adducirt. Die Streckung der Hand ist nur nach Bekämpfung eines fühlbaren Widerstandes möglich - losgelassen, nehmen die Hände die frïhere Stellung ein. Es besteht ferner sehr ausgesprochenes Chvostek'sches Phänomen. Beim 3 Jahre alten Kinde sind beide Fïsse in der charakteristischen Pes equinovarus-Stellung. - Nach Gebrauch von warmen Bädern und nach Darreichung von Bromkalium hörte der Symptomencomplex bei beiden in wenigen Tagen ganz auf.

Die uns interessirende Thatsache besteht in diesen Fällen in dem gleichzeitigen Auftreten der Tetanie bei Mutter und Kind; solche Fälle sind zwar schon mitgetheilt worden, aber wie ich aus mündlicher Mittheilung des Herrn v. Frankl-Hochwarth.entnommen habe, in geringer Zahl, weshalb ich dem Rathe des Collegen v. Frankl-Hochwarth folgend, die Fälle hiermit publicire.

Der dritte Fall von Tetanie, den ich dieses Jahr zu beobachten Gelegenheit hatte, entstammt der Nervenklinik des Prof. Laufenauer, dem ich für die gitige Ueberlassung des Krankheitsfalles hiermit meinen besten Dank ausspreche. Der Fall ist folgender:

Alexander $0 \ldots, 17$ Jahre alt, Sehuhmacher.

A n amnese. Vor 6 Monaten durchnässt bei einem Gewitter im Freien, begann er zu bemerken, dass beide Hände, Füisse und das Gesicht in 
Begleitung schwacher Schmerzen sich contracturirten. Die ganze Nacht dauerte die Contractur an, um Morgens zu schwinden. Er ging auch Nachmittags zur Arbeit. Bald stellte sich Kriebeln im Gesicht und den Extremitäten ein. Von dieser Zeit an litt er jede Woche an mehrstiundig dauernden Contracturen an den genannten Körpertheilen. Er gebrauchte spirituöse Einreibungen. - Nach einiger Zeit hörten diese Contracturen auf, um nach Monaten, nach einer neuerlichen Durchnässung wieder zu erscheinen. Seitdem traten diese Contracturen anfallsweise bis zu dem heutigen Tag anf. - Die Wohnung, die er bewohnte, war feucht.

Status praesens. Eltern und Geschwister leben und sind gesund. Die Grossmutter muitterlicherseits sieht nächtlich Thiere und Menschen; nachdem sie Licht gemacht, sieht sie dieselben nicht mehr; sie ist 70 Jahre alt. - Patient war ausser der jetzigen Erkrankung nie vorher krank.

Pupillen gleichweit, reagiren auf Licht und Accommodation prompt. In beiden Gesichtshälften deutliches Facialisphänomen; die leiseste Berührung des gemeinsamen Stammes des Facialis sowie dessen Ramus frontalis ist von einer lebhaften clonischen Zuckung begleitet. - Druck auf die übrigen Nervenstämme bleibt erfolglos. Beim plötzlichen Anfassen der Armmuskeln contrahiren sich dieselben lebhaft, so z. B. fasse ich den M. biceps mit zwei Fingern rasch an, so beugt sich der Unterarm; bei Anfassen oder auch Beklopfen der Extensoren des Unterarmes extendirt sich die Hand u. s. w. Die mechanische Erregbarkeit der Muskeln ist also lebhaft gesteigert (Erb-Symptom). Die idiomusculäre Wulstbildung ist sehr ausgesprochen. - Die mechanische Uebererregbarkeit erstreckt sich auf die Hals-, Riticken-, Brustkorb- und Armmusculatur, fehlt an den Unterextremitäten. Die Muskelbewegungen sind frei. Es besteht keine Ataxie, noch ist Romberg'sches Symptom nachweisbar.

Die Hautreflexe sind lebhaft. Kniephänomene sehr lebhaft. Sensibilität normal. Stereognostischer Sinn erhalten. - Für die vasomotorischen Verhältnisse ist als charakteristisch der Umstand hervorzuheben, dass die Stelle der Brustplatte, an welcher Vormittags $10 \mathrm{Uhr}$ die positive Elektrode angebracht war, noch um 4 Uhr Nachmittags stark geröthet erscheint, daselbst verspïrt Patient ein Prickeln. Die Haut zeigt beim Entblössen die bekannte Gänsehautbeschaffenheit.

Faradische Untersuchung ergiebt keine verwerthbaren positiven Resultate im Sinne einer erhöhten elektrischen Erregbarkeit. Die galvanische Untersuchung konnte äusserer Umstände wegen nicht galvanometrisch vorgenommen werden. Bei Reizung des M. triceps mittelst mittelstarken faradischen Stromes erhält man dessen tonische Contractur, welche noch einige Zeit nach Aufhören des Stromes anhält und dentlich fühl-und sichtbar bleibt. Dieses Symptom lässt sich an anderen Muskeln nicht nachweisen.

Sensorielle Uebererregbarkeit (Hoffmann'sches Symptom) liess sich für Auge und Ohr nicht nachweisen. -

Dieser Fall zeigt nun auch die klassischen Symptome der Tetanie. - Die intermittirend auftretenden tonischen Krämpfe in den Händen, sowie die gesteigerte mechanische Erregbarkeit der Muskeln und das Vorhandensein des Facialisphänomens lassen trotz Nichtunter- 
suchtsein der elektrischen Uebererregbarkeit und trotz Fehlens des Trousseau'schen Phänomens, die Diagnose mit Sicherheit auf Tetanie stellen. Hervorzuheben ftir wichtig erachte ich die myotonische Reaction, welche im M. triceps sehr dentlich nachzuweisen war, meines Wissens ist dieselbe bei Tetanie noch nicht beobachtet worden. Der andere Umstand, welchem wir unsere Aufmerksamkeit schenken wollen, ist derjenige, dass Patient als Beschättigung die eines Schusters angiebt; v. Frankl-Hochwarth hat bekanntlich für die Endemie in Wien die auffallend grosse Betheiligung von Personen dieses Gewerbes bei der Tetanie nachgewiesen. Ueber diesen Punkt weiter unten.

Auf Grund dieser meiner drei Beobachtangen blätterte ich in der Literatur der Tetanie nach und fand, dass so manche Punkte in der Aetiologie sowie Pathologie dieser Krankheit noch der Aufklärung bedürfen, so dass ich mich bewogen fühlte, theils auf Grund der bisherigen literarischen Angaben, theils durch Vergleich mit anderen Krankheiten auf diese Punkte näher einzugehen. Im Folgenden theile ich nun das Resultat meiner Vergleichungen mit, und hoffe ich hierdurch ein wenig zur Klärung einiger wichtiger Fragen beigetragen zu haben.

\section{Die Aetiologie der Tetanie.}

Nach einer einheitlichen ätiologischen Uisache fahnden wir umsonst. Es giebt Fälle, welche für die infectiöse Natur des Leidens sprechen, wieder andere, die auf einer Intoxication beruhen; endlich yiebt es auch solche, in denen gar keine ätiologische Ursache aufzufinden ist. Die infectiöse Natur des Leidens konnte weder bacteriologisch noch experimentell (v. J a k s ch) bisher nacbgewiesen werden. v. Frankl-Hochwarth regte die Aufmerksamkeit zuerst auf die infectiöse Natur der Tetanie - seine Gründe, die er für die Bestätigung aufführt, sind folgende: 1. „, das epidemisch-endemische Auftreten; 2. das häufige Einsetzen unter fieberhaften Erscheinungen ....; 3. das Erscheinen der Tetanie im Prodromalstadium und im Verlaufe typischer Infectionskrankheiten; 4. das Vorkommen ballucinatorischer Verworrenheit - in ähnlicher Weise wie bei anderen Infectionskrankheiten; 5. das gleichzeitige Vorkommen der Krankheit in mehreren Fällen in einer Familie oder in einem Hause (s. meine ersten zwei Fälle); 6. der Umstand, dass manche Jahre sehr viele, andere viel weniger Fälle bringen." - Alle diese Umstände sprechen auch nach meiner Ansicht scheinbar für die infectiöse Natur des Leidens andererseits lassen sich sehr viele Umstände gegen eine specifis che Infection anfibhren. 
Alle jene Tetaniefälle, welche nach Vergiftungen, nach Strumaexstirpation, im Verlaufe von Infectionskrankheiten, bei Rhachitis, Gastrektasie, Schwangerschaft und Säugen auftreten, sprechen entschieden gegen eine specifische Infection, hieraus folgt, dass wir für die Tetanie keinen specifischen Mikroorganismus verantwortlich machenkönnen, daes mit unserer Auffassung unvereinbar ist, dass eine Infectionskrankheit ausser dem specifischen Krankheitserreger noch andere hervorrufende Ursachen hätte. - Wir können also die Tetanie nicht als eine Infectionskrankheit sui generis betrachten. Wenn wir andererseits all die Zustände und Krankheiten berïcksichtigen, in deren Folge die Tetanie auftritt, so finden wir einen, allen gemeinsamen Zug in dem Umstande, dass sämmtliche obengenannten Krankeiten und Zustände eine allgemeine Ernährungstörung bedingen, und wir halten dafür, dass dieselbe für die Tetanie verantwortlich zu machen ist. Dies wollen wir nun des Näheren ausführen und begründen. -

- Es ist eine alte Erfahrungsthatsache, dass im Verlaufe der verschiedensten Infectionskrankheiten (Typhus, Scharlach, Diphtherie u. s. w., ich rechne auch die Malaria hierher, trotz des plasmodischen Ursprungs) die Tetanie sich entwickeln kann; in diesen Fällen ist es wohl berechtigt, einen Zusammenhang zwischen den beiden vorhandenen Krankheiten $z u$ vermuthen. Ich glaube, wir finden diesen $\mathrm{Zu}$ sammenhang, wenn wir nachweisen können, dass die verschiedensten Infectionskrankheiten das Nervensystem auch in Mitleidenschaft ziehen können und dasselbe in einen Zustand versetzen, welcher für die Entwicklung der Tetanie günstig ist. Bei der Beantwortung dieser Frage spielen die Tetanien, welche nach Intoxicationen anftreten, eine wichtige Rolle, so dass ich vorerst diese betrachte. Wir verfügen über recht viele Beobachtungen (Gowers, Purjesz), welche beweisen, dass die Tetanie bei Personen sich entwickeln kann, die an Vergiftungen mittelst chemischer Gifte leiden. Als solche Gifte sind bekannt Ergotin, Alkohol, Chloroform, Blei, Phosphor. In die Reihe der Intoxicationstetanien gehört auch die nach Strumaexstirpationen auftretende, da es sich nach der allgemein angenommenen Ansicht in diesen Fällen um die Vergiftung durch einen im Blute vorbandenen, jedoch durch das Fehlen der Schilddrüse nicht schadlos gemachten Toxin handelt. Aber es giebt noch eine Gruppe von Tetanien, welche hierher, in die Reihe der Intoxicationstetanien gehört und dies sind diejenigen Fälle, in welchen sich an eine Gastroektasie die Tetanie anschliesst. Nach Untersuchungen von B ouveret und Devie ist es ein, dem Brieger'schen Peptotoxin analoges Toxin, 
welches sich in diesen Fällen bildet; zur selben Gruppe gehören weiter die Tetanien nach Darmerkrankungen, bei diesen spielt auch ein, durch die Därme zur Aufsaugung kommendes Toxin nach allgemein angenommener Auffassung, die Rolle der Krankheitsursache. Alle diese verschiedenartigsten Vergiftungen sind im Stande, Tetanie hervorrufen; wir wollen nun versuchen, eine Erklärung für die gemeinsame Wirksamkeit dieser verschiedenen Gifte zu finden. Als Ausgangspunkt dienen die chemischen Gifte. Aeltere Untersuchungen von Pop off, Tschisch u. A. sprechen für das Vorhandensein von Ganglienzellenveränderungen bei den verschiedensten Vergiftungen. Kreyssig und Schultze jedoch bestritten die Resultate der oben genannten Forscher und erklärten die beschriebenen Veränderungen für Artefacta. Um die Frage nochmals zu prüfen, entschlossen wir uns, Schaffer und ich, im Jahre 1890 die Vergiftungsversuche wieder aufzunehmen, um so mehr, da uns eine neue Methode für Ganglienzellenfärbung zur Verfügung stand. Dies war die seitdem bekannte und allgemein anerkannte Methode des Dr. F. Nissl. Im Jahre 1891 war ich schon in der Lage, die Veränderungen der Ganglienzellen im Rückenmarke der Kaninchen nach Vergiftungen mit Phosphor und Morphium zu veröffentlichen; bald kamen die Untersuchungen Schaffer's zur Veröffentlichung, welche fur das Blei, Arsen, Antimon nachwiesen, dass dieselben Veränderungen in den Ganglienzellen hervorrufen. Später folgten die Untersuchungen von $\mathrm{P}$ a n d i über Cocain, Antipyrin mit ähnlichen positiven Resultaten. In meiner damaligen Publication „Ueber die Veränderungen der Ganglienzellen bei Phosphor- und Morphinvergiftung" hob ich hervor, dass ich die gefundenen Veränderangen nicht als specifisch für bestimmte Gifte anerkenne, da ich doch bei den zweiverschiedenen Vergiftungen dieselben Ganglienzellenveränderungen gefunden habe; ich schlossaus diesem Verhalten, dass diese Gifte eine allgemeine Ernährungsstörung. hervorrufen, als deren Resultat die Erkrankung der Ganglienzellen zu betrachten ist. Für diese Annabme betrachtete ich als Stütze diejenige Erfahrung, dass bei diesen Vergiftungen in den verschiedensten Organen krankhafte Veränderungen entstehen. S c h af fer schloss sich dieser meiner Auffassung an und dehnte sie auf die von ihm untersuchten Vergiftungen (Blei, Arsen, Antimon) auch aus. Bei all diesen Vergiftungen finden wir die ausgesprochensten Veränderungen in den Vorderhornganglienzellen des Rückenmarks, der Intensität der Vergiftung entsprechend stärker oder weniger stark ausgesprochen. Auf die Thatsache, dass eine partiell erkrankte Ganglienzelle noch func- 
tionsfähig sein könne, haben Friedmann und Schaffer die Aufmerksamkeit gelenkt. Auch müssen wir zugeben, dass kranke Ganglienzellen noch restitutionsfähig seien. In nächsten Kapitel werde ich die Gründe darlegen, welche dafür sprechen, dass die Tetanie aller Wahrscheinlichkeit nach, eine centrale Erkrankung ist. Hier muss ich es nur noch einmal betonen, dass für all die chemischen Gifte, welche die Tetanie hervorrufen können, dargethan ist, dass dieselben das Centralnervensystem angreifen, dass sie Ganglienzellenveränderungen hervorrufen und hiermit ist die Grundlage zur eventuellen Entwicklung einer Tetanie gegeben.

Wenn wir nun die Fälle von Tetanien betrachten, welche im Verlaufe infectiöser Erkrankungen auftreten, so liegt die Vermuthung nahe, die bei denselben sich entwickelnden Toxine, Ptomaïne, als den chemischen Giften analoge zu betrachten, denselben auch die Fähigkeit zuzuschreiben, gegebenen Falles, durch eine allgemeine Ernährungsstörung auch das Centralnervensystem in Mitleidenschaft zu ziehen und hierdureh zur Entwicklung der Tetanie die Grundlage abzugeben. Diese unsere Hypothese findet ferner ihre Stütze in der Beobachtung, lant welcher im Verlaufe oder im Gefolge infectiöser Erkrankungen (z. B. Typhus, Influenza u. s. w.) Erkrankungen des Centralnervensystems (Paraplegien, Incontinenta urinae et alvi, Myelitis u. s. w.) auftreten können; wir sehen, dass auch bei der Diphtherie nicht nur Erkrankungen der peripheren Nerven, sondern auch des Rückenmarks (Preisz) in Beobachtung kommen. Die Veränderungen des Centralnervensystems nach infectiösen Erkrankungen sind noch so wenig studirt, dass wir uns vorläufig mit diesen positiven Ergebnissen zufrieden geben müssen. Wenn wir weiter bedenken, dass die infectiösen Erkrankungen in den verschiedensten Geweben des Körpers Veränderungen bedingen, so z. B. die Veränderungen der Muskelsubstanz u. s. w., welche nicht direct specifischer Natur sind, sondern der Ausfluss einer Gesammternährungsstörung sind, so wird es uns nicht überraschen, auch in den nutritiven Vorgängen gegenüber sebr sensiblen Centralnervensystem Veränderungen zu finden. Diese Veränderungen müssen nicht destructiver Art sein, es ist die Möglichkeit wohl zu beachten, dass dem geänderten Chemismus entsprechend nur eine Functionsänderung sich merklich macht, welche sich dann, mit der Wiederkehr normaler Verhältnisse, ausgleicht. Unter denselben Gesichtspunkt fallen die Fälle von Tetanien nach Strumaexstirpation, sowie nach Gastrektasien und Darmerkrankungen. Das hypothetische Toxin bei Strumaexstirpation, sowie die Toxine bei Gastrektasien, Darmerkrankungen spielen meines Erachtens dieselbe Rolle bei der Hervorrufung der Tetanie, wie 
die angeführten Gifte. Dass irgend ein Gift bei diesen Fällen von Tetanie im Spiele ist, beweist auch der Verlauf solcher Tetanien. Sowohl die nach Strumaexstirpation als infolge von Gastrektasien auftretenden Tetanien sind langwierig und enden meistentheils tödtlich; die Ursache davon liegt eben in dem Umstande, dass die, die Tetanie hervorrufenden Toxine fortwährend producirt werden und dass es daher nicht nur zur Functionsänderung, sondern zum Functionsausfall, i. e. Tod kommen muss. Demgegenüber sind diejenigen Tetaniefälle, welche infolge von Vergiftungen oder in Verlaufe infectiöser Erkrankungen auftreten, von kurzer Dauer, der zu Grunde liegenden Erkrankung entsprechend. Natürlich sind auch Fälle von Tetanien nach Vergiftungen und infectiösen Erkrankungen zu finden, welche tödtlich verlaufen, da doch von der Intensität der Vergiftung resp. Infection es abhängt, ob es nur beim Functionsausfall sich bewendet oder ob derartige Veränderungen hervorgerufen werden, welche Functionsausfall bedingen. Die Resultate neuerlicher Beobachtungen, resp. Heilungsversuche, welche in der Darreichung von Thyreoideatabletten, resp. in der Implantation von Thyreoideadrüsen bestanden, wie sie Gotts te in ausgeführt hat, sprechen auch für die Toxintheorie.

Aus dem bisher Angefiuhrten folgt, dass eine grosse Anzahl von Tetanien ätiologisch in eine Gruppe gehört, welche dadurch charakterisirt ist, dass bei derselben Vergiftungen des Organismus die Hauptrolle spielen; die vergiftende Substanz umfasst nun chemische Gitte (Alkohol, Chloroform, Blei, Phosphor, Ergotin u. s. w.), Toxine (bei Strumaexstirpation, Gastrektasien, Darmerkrankungen), endlich Toxine, Ptomaïne von Bacterien (bei den verschiedensten infectiösen Erkrankungen). Für die in der Aetiologie der Tetanie vorkommenden chemischen Gifte (Ergotin, Phosphor, Plumbum u. s. w.) ist es erwiesen, dass dieselben im Centralnervensystem Veränderungen bewirken können; es liegt nahe, auf solcher Grundlage sich entwickelnde Tetanien pathologisch derart zu erklären, dass wir diesen Veränderungen im Centralnervensystem Rechnung tragen. Dasselbe gilt von den iibrigen zu dieser Gruppe vereinigten Tetanien: All diese Vergiftungen verursachen, unserer Auffassung gemäss, eine Ernährungsstörung des Organismus, an welcher Ernährungsstörung auch das Centralnervensystem mitbetheiligt und hierdurch für Tetanie die Grundlage geschaffen ist. Verfolgen wir nun eine andere Gruppe von Tetanien. Bei der Rhachitis, also einer Gesammternährungsstörung par excellence, findet man sehr oft die Tetanie. So sah S ze gö bei 205 rhachitischen Kindern 104 mal Tetaniesymptome. Szegö berichtet hierüber Folgendes: „Die für die Tetanie Erwachsener charakteristischen Symptome sind bei Kin- 
dern auch vorzufinden. Aber es erhellt eben aus friheren Untersuchungen, dass diese für die Tetanie so charakteristischen Symptome nicht mehr als charakteristisch betrachtet werden können bei der Tetanie der Kinder, da bei Rhachitischen das Facialisphänomen und das Trousseauphänomen sehr oft aufzufinden ist."

Dieser Zustand entspricht demjenigen, den v. Frankl-Hoch warth als tetanoiden bezeichnet hat. Nach unserer Meinung sprechen diese tetanoiden Zustände dafür, dass im Nervensystem eine Prädisposition für Tetaniesymptome existirt; dieser Zustand des Nervensystems ist der Rhachitis zuzuschreiben, um so mehr, als wir bei Kindern überhaupt einen sehr labilen Erregungszustand des Nervensystems antreffen, welcher doch von den Ernährungsstörungen, welche die Rhachitis zweifellos bedingt, sehr verändert werden kann und die Tetaniesymptome ermöglicht.

Es erübrigt noch, über zwei Zustände zu berichten, bei welchen auch Ernährungsstörungen sehr oft vorhanden sind und in welchen die Tetanie auch anzutreffen ist, das ist die Gravidität und das Säugen. Die bei diesen Zuständen tagtäglich wahrnehmbaren Gemüthsveränderungen, welche in schwereren Fällen in Cerebralerkrankungen sich kundgeben, dienen als Wegweiser dafür, wie empfindlich in diesen Zuständen das Centralnervensystem ist, und ich glaube, wir gehen nicht fehl, wenn wir in den Ernährungsstörungen die Ursache bei diesen auftretenden Tetanien im oben ausgefiuhrtem Sinne suchen.

Dem gegenüber steht jene Gruppe von Tetanien, in denen kein Gift aufzufinden ist, welche aber durch ihr epidemisches Auftreten charakterisirt sind. Auffallend ist bei diesen Epidemien das von v. Frankl. Hochwarth betonte Mehrbetheiligtsein von Leuten eines gewissen Gewerbes, namentlich der Schuster und der Schneider. Dies spricht auch entschieden gegen eine specifische Infection. Es ist möglich, dass 0 ppenheim Recht hat, indem er die Vermuthung ausspricht, dass im Leder irgend welche toxische Substanzen enthalten sind, die als Krankheitsursache zu betrachten seien. Warum werden aber die Schneider bevorzugt? Wenn diese Fälle von Tetanie infectiöser Natur sind, warum beschränkt sich der pathogene Mikroorganismus auf 17 Proc. Schneider? Es ist möglich, dass eine Aufklärung uber diesen Punkt uns erst eine in Zukunft erfolgende gründliche Anamnese betreffs irgend eines einwirkenden Giftes geben wird.

Als Ursache der Tetanie betrachten wir aiso in vielen Fällen eine allgemeine Ernährungsstörung, welche theils durch verschiedene Gifte (chemische Gifte, Toxin nach Strumaexstirpation, Peptotoxin, Darmtoxin) hervorgerufen wird, theils 
mit körperlichen Zuständen, wi e Gravidität, Säugen einhergeht, oder aber durcb eine den ganzen Organismus betreffende Krankheit, Rhachitis, bedingt ist.

Dem gegeniuber stehen jene Tetanien, welche in ihrer Gesammtbeit den Eindruck einer Infection machen; dass es einen speciellen Krankheitserreger geben soll, haben dic bisherigen Untersuchungen nicht gerechtfertigt. Es ist denkbar, dass auch für diese Gruppe die Rolle irgend eines Agens nachzuweisen sein wird, welches eine allgemeine Ernährungsstörung bedingend die Tetanie hervorruft - dann möchte selbstredend auch diese Gruppe mit der ersten vereinigt werden.

In dem folgenden Kapitel versuche ich den Nachweis zu fiuhren, dass die Tetanie eine centrale Erkrankung ist. Daselbst werde ich auch bestrebt sein, eine Erklärung daruber abzugeben, wie ich die Rolle der hier angeführten Ernährungsstörung bei der Hervorrufung der Tetanie mir vorstelle.

\section{Pathologie der Tetanie.}

Ueber den Sitz der Erkrankung stehen zwei entgegengesetzte Meinungen einander gegentiber. Ein Theil der Forseber sucht denselben im peripherischen Nervensystem, während ein anderer Theil das Centralnervensystem als Ausgangspunkt der Symptome bezeichnet. Die musculäre Theorie ist wohl von sämmtlichen Autoren verlassen. - Im Vorhergehenden haben wir schon betont, dass anch wir den Sitz der Erkrankung im Centralnervensystem zu finden glauben. Bevor ich nun die Gründe hierfür anführe, erlaube ich mir einige biologischphysiologische Thatsachen zu vermerken. Nach Ansicht älterer Autoren verdankt das Nervensystem zwei von einander unabhängigen Gebilden seine Function: den Zellen und den Fasern. Die mit der Golgi'schen Methode gewonnenen Resultate erweisen unzweidentig, dass eine jede Nervenfaser ihren Ursprung einer Zelle verdankt, demnach sind dieselben als eine Einheit aufzufassen; trotz alledem besteht sowohl chemisch wie morphologisch eine Differenz zwischen diesen zwei Gebilden. Es ist zwar richtig, dass bei den niederen Thieren dieser Unterschied nicht aufzufinden ist, derselbe wird aber mit der steten Differenzirung bei Höherstehenden immer deutlicher und ist bei den Vertebraten am ausgesprochensten. - Die Ganglienzelle unterscheidet sich von der Nervenfaser chemisch, indem die Proteïnsubstanz viel mehr Wasser enthält, als dieselbe Substanz der Fasern; ferner besteht ein grosser Unterschied in der Blutversorgung, die Ganglienzellen communiciren fast direct mit den Blutgefässen; an ersteren ist keine Grenzmembran sichtbar; während die Fasern durch 
die Myelinscheide geschutzt sind; ferner ist die graue Substanz des Centralnervensystems viel blutreicher als die weisse. Auch morphologisch besteht ein Unterschied.

Die mittelst der Nissl'schen Methode gewonnenen Bilder zeigen, dass die färbbare Substanz, welche den Zellenleib ausfüllt, nicht in den Axencylinder verfolgbar ist, aus welchem die periphere Nervenfaser entsteht (S chaffer, B enda). Alle diese Differenzen weisen darauf hin, dass auch functionell ein Unterschied vorhanden ist. Es ist eine allgemein anerkannte Auffassung, dass die Zelle als Reizaufnehmerin und als Reizauslösende zu betrachten ist, während die Faser die Leitung des Reizes besorgt. Die physiologische Erfahrung, dass, je weiter vom Muskel der Nerv gereizt wird, desto grösser der Erfolg der Muskelzusammenziehung ist, spricht dafür, dass die Fasern nicht nur Leiter von Reizen sind, sondern auch zur Verstärkung derselben beitragen können. Trotzdem besteht die Hauptaufgabe der Faser in der Fortleitung des Reizes, dies erbellt aus den folgenden Erörterungen und Thatsachen, welche ich nach Spencer mittheile. Jeden Nervenreiz müssen wir als eine Bewegung auffassen. Dieser Nervenreiz resp. diese Bewegung wird durch moleculare Bewegung weiterbefördert. Wir wissen, dass die moleculare Bewegung eines zusammengesetzten Körpers in zweierlei Weise vor sich gehen kann: 1. einige oder sämmtliche Bestandtheile zersetzen sich und vertheilen sich in der Umgebung allein oder in Form neuer Verbindungen; 2. die Grundbestandtheile verändern ihre Lage gegenseitig so, dass die wahrnehmbaren Eigenschaften der Materie sich ändern, jedoch ohne eine Aenderung ihrer physikalischen Beschaffenheit. Die erste Art bezeichnen wir als Zersetzung, die zweite als isomerische Umwandlung. Die Zersetzung erfolgt mit Freiwerden von Bewegung, während bei der isomerischen Umwandlung das Freiwerden oder das Aufnehmen von Bewegung sehr wuerheblich ist. Die Zersetzung durchmachenden Bestandtheile der Materie nehmen ihre frühere Anordnung nur sehr schwer oder gar nicht mehr auf; bei der isomerischen Umwandlung erfolgt die Wiederaufnahme der früheren Anordnung schon auf kleine Veranlassungen. Diese zwei entgegengesetzten Arten der Molecularbewegung sind auch wahrscheinlich bei den beiden Componenten des Nervensystems vorhanden; während die Zellen die Zersetzung durchmachen, geht in den Nervenfasern die isomerische Umwandlung vor sich; dementsprechend erfolgen in den Zellen zersetzende Molecularbewegungen und hiermit Freimachen von Bewegung, während die Substanz der Nervenfasern nur solche Veränderungen erleidet, welche weder mit Zersetzung, noch mit Freiwerden von Bewegung 
einhergeht. Diese bei Herbert Spencer auffindbare Hypothese erscheint durch all die Differenzen morphologiseher und chemischer Art, welche wir im Vorhergehenden zwischen Nervenzelle und Nervenfaser besprochen haben, gestiutzt zu sein. Die graue Substanz erhält mehr Wasser als die weisse, letztere enthält 12 Theile Wasser und 100 Theile feste Substanz; bei der letzteren ist das Verhältniss wie 35:100. Wasserreichthum ist aber eine Erforderniss für Molecularbewegungen und kommt in denjenigen Substanzen vor, in welchen die Veränderungen lebhaft sich vollziehen (Spencer). Daraus folgt, dass die graue Substanz, id est die Nervenzelle, lebbaftere Veränderungen aufweist, als die weisse.

Aus alledem geht hervor, dass die Rolle der Nervenfasern hauptsächlich in der Fortleitung der Bewegungen besteht und nur zum geringeren Theil in deren Verstärkung, während die von ihnen den Ganglienzellen zugeleiteten Bewegungen (Erregungen) daselbst schon grosse Molecularveränderungen hervorrufen, dessen Ausdruck der hervorgerufene Effect, z. B. die Muskelzusammenziehung, ist. - In der uns jetzt interessirenden Frage, welcher Theil des Nervensystems bei der Tetanie in Mitleidenschaft gezogen ist, spielt das hier Vorgetragene eine wichtige Rolle. Die schon auf geringfügigen Reiz erfolgenden ausgiebigen Muskelbewegungen, welche wir in dieser Krankheit zu beobachten Gelegenheit haben, sprechen dafür, dass wir den Sitz der Erkrankung in den Zellen suchen, von denen wir wissen, dass sie die Eigenschaft von Freimachen der Bewegungen in grossem Maasse besitzen. Weiter verdienen folgende allgemeine Betrachtungen Beachtung. Sämmtliche Muskeln des Körpers sind fortwährend in gewissem Gleichgewichtszustand; wir besitzen keinen Muskel, welcher auch nur für einen Moment nicht in gewissem Spannungszustand wäre - das ist, was wir gemeinhin als Tonus der Musculatur bezeichnen. Nach allgemein angenommener Ansicht wird dieser Tonus von den Vorderhornganglienzellen aufrecht erhalten. Dies beweist jener Umstand, dass bei allen Erkrankungen des Rückenmarkes, bei welchen die Vorderhornganglienzellen zu Grunde gehen, dieser Tonus aufhört, die Muskeln werden schlaff. Andererseits nehmen wir doch an, dass das Gehirn mittelst der Pyramidenbahn auf diese Vorderhornzellen Einfluss austibt; indem bei Erkrankung resp. Degeneration der Pyramidenbahn es zur "Hyperfunction" dieser Zellen kommt, der Tonus nimmt zu. Wenn wir bei der Tetanie das Hauptsymptom betrachten, so sehen wir, dass dasselbe in intermittirenden tonisehen Contracturen besteht, in denselben erblicken wir nur eine Erhöhung der normalen Tonusverhältnisse. Dass der Grund nicht in den Muskeln liegt, ist 
wohl ausser Zweifel, dass er aber auch nicht im peripherischen Nervensystem zu suchen ist, dies beweisen wohl die angeführten Thatsachen. Ferneren indirecten Beweis dafür, dass die Zellen es sind, welche bei Tetanie die Symptome bedingen, sehen wir in dem Umstand, dass bei den Vergiftungen, in deren Folge die Tetanie auftritt, gerade diese motorischen Ganglienzellen des Rückenmarkes angegriffen sind. Es muss zwar bemerkt werden, dass alle die Gifte auch das periphere Nervensystem angreifen, und somit können wir dessen Mitwirkung bei Hervorrufung der Tetaniesymptome nicht mit aller erforderlichen Sicherheit ausschliessen, jedoch wenn wir das oben Auseinandergesetzte beachten, so ist es folgerichtig, die auch unter normalen Verhältnissen in grossem Maasse Bewegung freimachende Ganglienzellen eher für den Sitz des Hypertonus verantwortlich za machen, als die nur den Bewegungsimpuls fortleitenden Nervenfasern. - In dem Kapitel der Aetiologie erwähnte ich, dass die Wirkung der Intoxicationen in der Weise aufzufassen sei, dass dieselben eine allgemeine Ernährungsstörung hervorrufend, auch das Nervensystem angreifen und dessen Function verändern. Vielleicht nähern wir uns etwas der Wirklichkeit, wenn wir das Folgende beachten. Wir können sagen, dass es kein Organ giebt, welches bei Intoxicationen nicht angegriffen und in seiner Function nicht veränderlich wäre; um nur ein Beispiel anzufübren, sehen wir doch bei der Bleivergiftung die Störungen verschiedenster Organe (Darmkolik, Nephritis, Encephalopathie, Radialislähmung u. s. w.). - Dieser Umstand fordert nun auf, einen solchen Weg für die intoxicirende Substanz zu suchen, welcher in jedem Organ auffindbar ist, und dieses kann nur die Lymph-, resp. Blutbahn sein. Wenn wir nun annehmen, dass das Gift in die Blutbahn kommt, so kann es z. B. seine deletäre Wirkung in der Veränderung der Blutbeschaffenheit äussern. Nehmen wir z. B. an, dass es das Oxyhämoglobin angreift und zersetzt, hierdurch die Qualität des Blutes ändernd, leistet es die Möglichkeit zu der Erkrankung der weniger widerstandsfähigen Organe. Zur normalen Thätigkeit der Ganglienzellen ist die normale Blutbeschaffenheit erforderlich, dies erhellt auch aus dem Blutreichthum dieser Gebilde. Wenn nun diese Blutbeschaffenheit durch das Gift in obiger oder in irgend einer anderen denkbaren Weise verändert ist, so muss auch der Chemismus der Ganglienzelle hierunter leiden, dessen Ausdruck die veränderte Function sein wird. Das durch die Vergiftung angegriffene Organ braucht nicht functionsunfähig zu werden, dies wird nur dann der Fall sein, wenn die Vergiftung sehr intensiv ist, wenn ihre deletäre Wirkung sich auf einmal kundgiebt. Langsam einwirkende Gifte werden nur 
eine Functionsänderung hervorrufen; wenn aber die Vergiftung lange Zeit hindurch ununterbrochen fortbesteht, so tritt an Stelle der Functionsänderung der totale Functionsausfall, id est der Tod. - Schöne Beispiele liefern hierfür diejenigen Tetaniefälle, welche nach Strumaexstirpation (oder z. B. nach Gastrektasien) auftreten; hier handelt es sich nach unserer Meinung auch um eine Vergiftung, welche aber andauernd ist, weshalb sich zum im Anfang vorhandenen Functionsausfall der Zellen deren Functionslosigkeit resp. Tod anschliesst.

Dementsprechend miissen wir in solchen Fällen Veränderungen im Centralnervensystem resp. in den Zellen finden. Wir verfügen auch ubber Sectionen, welche in dieser Richtung positive Resultate ergaben, dies sind die Fälle von Weiss. Leider waren mir die Untersuchungen Weiss' im Original nicht zugänglich, deshalb muss ich mich darauf beschränken, was bei v. Frankl-Hochwarth und $\mathrm{H}$ of fmann berichtet ist. Weis s beschreibt die verschiedensten Veränderungen namentlich der Vorderhornganglienzellen des Riuckenmarkes. Seine Angaben sind von Vielen in Zweifel gezogen. Es ist wahr, dass die mit den älteren Methoden gewonnenen Resultate zweifelhafter Natur sein können, jedoch giebt mir der Umstand Anlass zum Bedenken, dass auch die positiven, mit älteren Methoden gewonnenen Resultate bei Vergiftungen, von Autoren (Kreyssig, Schultze) angezweifelt worden sind, während die neneren Untersuchungen die Richtigkeit der älteren Befunde in Vielem wieder bestätigen konnten. Demnach halten wir die Resultate $W$ eiss, wenn auch nicht für vollgültig, jedoch immer gewichtig genug, um in $\mathrm{Be}$ tracht gezogen zu werden. Die Frage könnte selbstredend in positiver Weise, nur durch Untersuchungen gelöst werden, welche mittelst den neuen Methoden gemacht worden sind. - Die Hypothese We is s', dass der Sympathicus für die Symptome verantwortlich gemacht werden muss, ist schon von anderer Seite grtindlich widerlegt, so dass ich hiervon absehe.

Dies sind die physiologiseh-pathologisch und pathologiseh-anatomischen Gründe, welche dafür sprechen, dass die Tetanie centralen Ursprunges sei, ich könnte dieselben noch mit jenem negativen Beweis erhärten, dass in den peripheren Nerven noch von keinem Autor Veränderungen angetroffen worden sind.

Gehen wir nun über zu den klinischen Symptomen der Tetanie; sehen wir, ob dieselben den centralen oder peripheren Charakter des Leidens an sich tragen. Vor Allem sind Symptome vorhanden, welche nur mittelst der Annahme einer Centralerkrankung erklärt werden können, wie dies tibrigens schon v. Frankl-Hochwarth hervorhebt. 
Diese sind: die Bilateralität der Symptome; ferner der von $\nabla$. FranklHochwarth beobachtete Umstand, dass Druck auf einen Nervenstamm die Contractur auch der Extremität der entgegengesetzten Seite zur. Folge hat, dieses Verhalten können wir nur mittelst der centralen Erregungsübertragung erklären. Nur centralen Ursprungs kann ferner der manchmal vorhandene Fussclonus sein. Der Sitz der Erkrankung kann nicht nur das Rückenmark, sondern auch die Oblongata und das Gehirn sein. Für die Betheiligung der Oblongata sprechen die vasomotorischen Erscheinungen (v. Frankl-Hochwarth). Der Antheil des Grosshirns prägt sich in denjenigen Fällen aus, in welchen Bewusstseinsstörungen, hallucinatorische Verwirrtheit u. s. w. vorhanden sind.

Ferner weist auf einen centralen Ursprung jener Umstand hin, dass sich Epilepsie an die Tetanie anschliessen kann, letztere ist wohl entschieden centralen Ursprungs. Die Trousseau-, Erb-, Hoffmann'schen Phänomene erhalten alle genügende Erklärung durch die Voraussetzung einer centralen Uebererregbarkeit. Schwerer sind die sensiblen Erscheinungen zu deuten - die Parästhesien, welche die Tetaniesymptome oft einleiten und auch als alleinige Symptome, als Aequivalente der Krampfanfälle beobachtet werden können. Verständlich sind aber dieselben auch bei centralem Sitz des Leidens, denn es lassen sich Belege dafür anführen, dieselben als centrale $\mathrm{Er}$ regungen mit peripherer Projection aufaufassen. Die während der Krampfanfälle voŕhandenen Schmerzen rechne ich nicht hierher, dieselben verdanken ibren Ursprung den durch die Muskelzusammenziehung erregten peripheren Nervenendigungen; ich spreche hier nur von den Parästhesien, welche die Anfälle einleiten oder sie überdauern. Das solche Parästhesien centralen Ursprunges sein können, beweist der Umstand, dass wir dieselben bei der mit der Tetanie oft sich vergesellschaftenden Epilepsie antreffen und als Aura kennen, an deren centralem Ursprung wohl kein Zweifel besteht. Aber wir sehen bei einem anderen centralen Leiden auch diese Parästhesien, d. i. bei der Syringomyelie, welch letztere Erkrankung uns den ferneren Beweis liefert, dass trophische Störungen, wie solche bei der Tetanie auch zur Benbachtung kommen (Haarausfall, Nägelwechsel), centralen Ursprunges sein können.

Ich will noch ein paar Worte über das Facialisphänomen sagen. v. Frankl-Hoch wart $\mathrm{h}$ wies dasselbe bei jungen nicht nervenkranken Individuen in 3-4 Proc. der Fälle nach; dies beweist, das bei sonst normalem Verhalten des Nervensystems eine Uebererregbarkeit des centralen Nervensystems bestehen kann, welche dieses Symptom bedingt. 
Dass eine Prädisposition für Tetanie bei exquisit centralen Leiden vorkommt, dies beweisen die Beobachtungen an Hysterischen und Neurasthenischen. Wir sehen bei beiden Erkrankungen das Auftreten des Chvostek'schen Phänomens. Die hysterischen Contracturen können den tetanischen vollkommen analog sein; es ist bekannt, dass gesteigerte elektrische und mechanische Erregbarkeit bei der Hysterie vorkommt; die Hyperästhesie der Sinne (Hoffmann'sches Symptom) ist bei beiden Leiden sehr oft anzutreffen.

Symptomengleichheit besteht zwischen dem Tic convulsif und dem Facialisphänomen, mit dem Unterschiede, dass ersterer spontan, letzteres auf peripheren Reiz erfolgt. Für die centrale Natur des Tic sprechen die psychischen Störungen, die gleichzeitig vorhanden sind. - La ufenauer und Högyes zeigten an hypnotisirten Hysterischen, dass der Druck gewisser Nervenpunkte in den entsprechenden Muskeln eine Contractur auslöst - dies ist ein mit der mechanischen Muskelnerven-Uebererregbarkeit der Tetanie analoges Symptom. Dies spricht wohl auch für ein centrales Entstehen der Tetaniesymptome.

(Nebenbei will ich bemerken, dass trotz der Symptomengleichheit der mechanischen Muskel- und Nervenerregbarkeit mit denen an hypnotisirten Hysterischen hervorzurufenden, ich den Mechanismus der beiden für versehieden halte; während ich bei der Hysterie die Uebererregbarkeit der motorischen Ganglienzellen durch Ausfall der Hemmung des Gehirneinflusses mir erkläre, ist diese Uebererregbarkeit bei der Tetanie durch die veränderten Ernährungsverbältnisse bedingt.)

Die von mir gemachte Beobachtung des faradischen Tetanus ist, wie wir es seit Erb's Untersuchungen wissen, ein Hauptsymptom der Thomsen'schen Krankheit, welche als hereditäre resp. familiäre Erkrankung wohl mit einem anormalen Zustande des Centralnervensystems zusammenhängt. Diese Coincidenz der Symptome, bei einem exquisit centralen Leiden, wie die Thomsen'sche Krankheit und der Tetanie, spricht auch für die centrale Natur der letzteren.

Bisher sprachen wir immer von einem centralen Sitz des Leidens, ohne näher im Centralnervensystem zu localisiren. Die auf S. 251 angefibhrten Thatsachen sprechen daftir, dass wir in den motorischen Ganglienzellen den Sitz für die motorischen Symptome verlegen, hierher gehören die intermittirend auftretende tonischen Muskelkrämpfe, das Chvostek'sche Phänomen, die mechanische und elektrische Uebererregbarkeit. Es giebt aber einen Symptomencomplex, dessen Ursprungsstelle anderweitig gelegen sein muss, das ist die Uebererregbarkeit der Sinne (Hoffmann'sches Symptom), die Parästhesien und die 
trophischen Störungen. Diese Symptome sprechen dafür, dass nicht nur die motorischen, sondern auch die sensiblen, resp. trophischen Nervenzellen in Mitleidenschaft gezogen werden können. - Endlich sprechen die psychischen Symptome für das Betheiligtsein der Corticalzellen. Wir ersehen hieraus, dass das ganze Centralnervensystem bei der Tetanie im Spiele sein kann; warum die Erkrankung einmal nur auf Rückenmarkssymptome beschränkt bleibt, warum ein andermal auch medullare, cerebrale Symptome vorhanden sind, können wir nicht beantworten.

Alles dieseinzeln und im Ganzenspricht dafür, dass wir die Tetanie als eine centrale Zellenerkrankung auffassen. In Betracht gezogen, dass bei der Annahme einer peripheren Erkrankung viele Symptome unerklärt bleiben, während mittelst der Annahme einer centralen, sämmtliche Symptome genügende Erklärung finden, neigen wir der Ansicht $z \mathfrak{u}$, dass die Tetanie eine centrale Erkrankung sei, wie dies übrigens schon $\mathrm{Erb}$ und $\mathrm{H}$ off $\mathrm{m}$ a $\mathrm{n}$ dargelegt haben. Kahler und $\mathrm{Neusser}$ halten dafür, dass bei der Tetanie das gesammte Nervensystem mitbetheiligt sei, Nothnagel nimmt auch diesen Standpunkt ein, jedoch hebt er, wie wir dies von v. FranklHochwarth erfahren, das Mitbetroffensein des peripheren Theiles hervor. Demgegenüber hält Schultze an der peripheren Natur des Leidens fest. - Wenn wir auch die Rolle der peripheren Nerven nicht ganz ausschliessen können, so sind wir in Anbetracht des Angeführten eher geneigt, ein primäres Betheiligtsein des Centralnervensystems anzunehmen und die Möglichkeit der mit ihm in Zusammenhang stehenden consecutiven, secundären Betheiligung der peripheren Nerven zuzugeben. -

\section{Literatur.}

v. Frankl-Hochwarth, Die Tetanie. Berlin 1891. (Reiches Literaturverzeichniss.) Daselbst die Angaben von v. Jaksch, Weiss, Kahler, Neusser, Nothnagel.

Pur jesz Zsigm ond, Lehrbuch der inneren Krankheiten. Artikel Tetanie. Ungarisch.

Popoff, Ueber Veränderungen im Rückenmark nach Vergiftung mit $\mathrm{As}, \mathrm{Pb}$ und Hg. Virchow's Archiv. Bd. XCIII.

Tschisch, Ueber Veränderungen des Rüekenmarks bei Vergiftung mit Morphium, Atropin, Silbernitrat, Alaunbromid. Ebenda. Bd. C.率

Kreyssig, Ueber die Beschaffenheit des Rückenmarks bei Kaninchen und Hunden nach Phosphor und Arsenikvergiftung nebst u. s. w. Ebenda. Bd. CII.

S ar bó, Die normale Structur der Ganglienzellen, des Kaninchenrückenmarkes und über deren Verănderungen nach Phosphor u. Morphinvergiftung. Ung. Arch. 1892. 
Schaffer, Ueber Veränderungen der Ganglienzellen nach Vergiftung mit $\mathrm{Pb}, \mathrm{As}$ und At. Ung. Archiv. 1893.

Pándi, Ueber Veränderungen der Ganglienzellen nach Vergiftung mit Brom,. Cocain, Nicotin und Antipyrin. Ebenda. 1893.

Gottstein, Versuche zur Heilung der Tetanie. Deutsche Zeitschrift für Nervenheilkunde. 1895. 3. u. 4. Heft.

Preis z, Beiträge zur Auatomie der diphth. Lähmungen. Ebenda. 1894. Nr. 1 u. 2. Sze gö, Ueber d. nervösen Erscheinungen d. Rhachitis (ungarisch). Ung. Arch. 1894. oppenheim, Lehrbuch der Nervenkrankheiten. Artikel Tetanie.

Herbert Spencer, Die Principien der Psychologie. Deutsch von Vetter. 1882. Ben da, Ueber die Bedeutung der durch basische Anilinfarben darstellbaren Nervenzellenstructuren. Neurolog. Centralbl. 1895. Nr. 17.

L a u fena uer, Högyes, Sitzungsberichte des königl. ung. Aerztevereins in Budapest in Orvosi Hetilap. 1894. 